\title{
Pacific
}

Journal of

Mathematics

\section{CLASSIFYING 3- AND 4-DIMENSIONAL HOMOGENEOUS RIEMANNIAN MANIFOLDS BY CARTAN TRIPLES}

VICTOR PATRANGENARU 


\title{
CLASSIFYING 3 AND 4 DIMENSIONAL HOMOGENEOUS RIEMANNIAN MANIFOLDS BY CARTAN TRIPLES
}

\author{
Victor PATRANGENARU
}

In this paper, we show how to use the method of Cartan triples (see V. Patrangenaru) in small dimensions.

We classify the 3-dimensional simply connected homogeneous Riemannian spaces, and the 4-dimensional simply connected homogeneous Riemannian spaces with 5-dimensional total isometry group. We show that the smallest dimension where locally homogeneous Riemannian manifolds that are not locally isometric to homogeneous Riemannian spaces exist, is 5 .

Acknowledgement. The first two sections cover in part the author's work at the University of Haifa. I wish to express my gratitude to my advisor, Professor Izu Vaisman.

\section{Preliminaries.}

The long history of homogeneous Riemannian spaces (on which we do not dwell here), knew an important moment with the appearance of É. Cartan's method of adapted frames [3, Chap XII] which was subsequently used by Ishihara [4] and Jensen [5] to list homogeneous Riemannian spaces of dimension four. In [13], we have shown that this method may be put in an algebraic form, called the method of Cartan triples, which provides us with necessary and sufficient conditions of local isometry of two locally homogeneous Riemannian spaces (l.h.R.s.).

The aim of the present paper is to use the formalism of Cartan triples in dimensions 3 and 4, in order to parametrize the isometry classes of simply connected homogeneous Riemannian spaces. We use and enhance the well known list of Milnor [9], of Riemannian curvatures of left invariant metrics on 3-dimensional Lie groups. We are led to a different proof of Thurston's [16] and part of Wall's [17] classification of 3 and 4 geometries.

Let $\mathfrak{g}$ be a Lie subalgebra of $\mathfrak{o}(\mathrm{n})$. We shall denote by $\mathfrak{g}^{\perp}$ the orthocomplement of $\mathfrak{g}$ with respect to the Killing form.

Let $\mathfrak{a}$ be a vector subspace of $\mathfrak{o}(n)$, such that $[\mathfrak{g}, \mathfrak{a}] \subseteq \mathfrak{a}$ and let $\Phi$ be an $\mathfrak{a}$-valued, k-multilinear map of $\mathbb{R}^{n}$. We say that $\Phi$ is $\mathfrak{g}$-invariant if for any 
$\xi \in \mathfrak{g}, X_{i} \in \mathbb{R}^{n}, i=\overline{1, k}$

$$
\left[\xi, \phi\left(X_{1}, . ., X_{k}\right)\right]=\sum_{i=1}^{k} \phi\left(X_{1}, . ., \xi\left(X_{i}\right), . ., X_{k}\right) .
$$

If the linear map $\Gamma: \mathbb{R}^{n} \rightarrow \mathfrak{g}^{\perp}$ and the bilinear map $\bar{\Omega}: \mathbb{R}^{n} \times \mathbb{R}^{n} \rightarrow \mathfrak{g}$ are $\mathfrak{g}$-invariant and if $\xi_{\mathfrak{g}}$ is the orthogonal projection (with respect to the Killing form) of $\xi \in \mathfrak{o}(n)$ on $\mathfrak{g}$, we say that $\mathrm{T}: \mathbb{R}^{n} \times \mathbb{R}^{n} \rightarrow \mathbb{R}^{n}$ respectively $\tilde{\Omega}: \mathbb{R}^{n} \times \mathbb{R}^{n} \rightarrow \mathfrak{g}$, defined by

$$
\begin{gathered}
T(X, Y)=\Gamma(Y) X-\Gamma(X) Y \\
\tilde{\Omega}(X, Y)=\bar{\Omega}(X, Y)-[\Gamma(X), \Gamma(Y)]_{\mathfrak{g}}
\end{gathered}
$$

are the $A-S$ (Ambrose - Singer) torsion respectively curvature associated to the triple $(\mathfrak{g}, \Gamma, \bar{\Omega})[13, \S 1]$ (one may try to find the connection with the infinitesimal model $[\mathbf{8}, \mathbf{1 1}, \mathbf{1 2}, \mathbf{1 5}]$ etc.).

An $n$-dimensional Cartan triple $[\mathbf{1 3}, \S 1]$ is a triple $(\mathfrak{g}, \Gamma, \bar{\Omega})$, where $\mathfrak{g}$ is a Lie subalgebra of $\mathfrak{o}(n), \Gamma: \mathbb{R}^{n} \rightarrow \mathfrak{g}^{\perp}$ is a linear map and $\bar{\Omega}: \mathbb{R}^{n} \times \mathbb{R}^{n} \rightarrow \mathfrak{g}$ is a bilinear skew symmetric map, which are both $\mathfrak{g}$-invariant and together with their associated $A-S$ torsion and curvature, satisfy the following identities:

$$
\sum_{\substack{\text { cycl } \\ X, Y, Z}} \tilde{\Omega}(T(X, Y), Z)=0, \quad \forall X, Y, Z \in \mathbb{R}^{n}
$$

$$
\sum_{\substack{\text { cycl } \\ X, Y, Z}}(T(T(X, Y), Z)-\tilde{\Omega}(X, Y)(Z))=0, \forall X, Y, Z \in \mathbb{R}^{n} .
$$

To the Cartan triple $(\mathfrak{g}, \Gamma, \bar{\Omega})$, one associates the Lie algebra $\mathfrak{k}(\mathfrak{g}, \Gamma, \bar{\Omega})=$ $\left(\mathfrak{g} \oplus \mathbb{R}^{n},[],\right)$ with [ , ] defined by:

$$
\begin{gathered}
{[\xi, \eta]=[\xi, \eta] \quad \forall \xi, \quad \forall \eta \in \mathfrak{g}} \\
{[\xi, X]=\xi X \quad \forall \xi \in \mathfrak{g}, \quad \forall X \in \mathbb{R}^{n}} \\
{[X, Y]=-T(X, Y)-\tilde{\Omega}(X, Y), \quad \forall X, Y \in \mathbb{R}^{n} .}
\end{gathered}
$$

In the upper formulas, $[$,$] is the commutator of two matrices, and X$ is seen as a column matrix, such that $\xi X$ is the ordinary multiplication of matrices; obviously $\mathfrak{g}$ is a Lie subalgebra of $\mathfrak{k}(\mathfrak{g}, \Gamma, \bar{\Omega})$. Let $K$ be the simply connected Lie group of Lie algebra $\mathfrak{k}(\mathfrak{g}, \Gamma, \bar{\Omega})$, and let $G$ be the connected Lie subgroup of $K$ of Lie algebra $\mathfrak{g}$. A Cartan triple is closed if $G$ is closed in $K$ [13, Remark 2.1]. 
Let $\mathbf{C}=(\mathfrak{g}, \Gamma, \bar{\Omega}), \mathbf{C}^{\prime}=\left(\mathfrak{g}^{\prime}, \Gamma^{\prime}, \bar{\Omega}^{\prime}\right)$ be n-dimensional Cartan triples; we say that $\mathbf{C}$ is smaller than $\mathbf{C}^{\prime}$, if $\mathfrak{g} \subseteq \mathfrak{g}^{\prime}$, and with respect to the decompositions $\mathfrak{g}^{\prime}=\mathfrak{g} \oplus \mathfrak{a}, \mathfrak{g}^{\perp}=\left(\mathfrak{g}^{\prime}\right)^{\perp} \oplus \mathfrak{a}$, we have

$$
\bar{\Omega}^{\prime}=\bar{\Omega} \oplus \bar{\Omega}_{\mathfrak{a}}, \quad \Gamma=\Gamma^{\prime} \oplus \Gamma_{\mathfrak{a}},
$$

where $\bar{\Omega}_{a}^{\prime}, \Gamma_{\mathfrak{a}}$ are the a-components of $\bar{\Omega}^{\prime}, \Gamma$ with respect to the decompositions of $\mathfrak{g}^{\prime}, \mathfrak{g}^{\perp}$ considered above.

$$
\bar{\Omega}_{\mathfrak{a}}^{\prime}(X, Y)=[\Gamma(X), \Gamma(Y)]_{\mathfrak{a}}-\Gamma_{\mathfrak{a}}(\Gamma(X)(Y)-\Gamma(Y)(X)) .
$$

Let $M_{n}$ be the set of maximal Cartan triples. The action $A_{n}$ of $O(n)$ on the set $C_{n}$ of n-dimensional Cartan triples [13, Proposition 3.2], defined by: $A_{n}((\mathfrak{g}, \Gamma, \bar{\Omega}), a)=\left(\mathfrak{g}^{\prime}, \Gamma^{\prime}, \bar{\Omega}^{\prime}\right)$, where

$$
\begin{gathered}
\mathfrak{g}^{\prime}=\operatorname{Ad}\left(a^{-1}\right) \mathfrak{g} \\
\Gamma^{\prime}(\cdot)=\operatorname{Ad}\left(a^{-1}\right) \Gamma(a(\cdot)) \\
\bar{\Omega}^{\prime}(\cdot, \cdot)=\operatorname{Ad}\left(a^{-1}\right) \bar{\Omega}(a(\cdot), a(\cdot))
\end{gathered}
$$

leaves $M_{n}$ invariant.

We showed in [13, Theorem 3.1. a)] that there is a bijection from the set of local isometry classes of $n$-dimensional l.h.R.s.-es and the set of $A_{n}$ orbits of $M_{n}$. Under this map homogeneous Riemannian spaces correspond to orbits of closed Cartan triples.

A l.h.R.s. corresponding by the inverse of this map to a Cartan triple $\mathbf{C}$, is called a local geometric realization of $\mathbf{C}$. If $\mathbf{C}$ is closed, there exists a unique, up to an isometry, simply connected Riemannian homogeneous space $\mathbf{M}$ corresponding to $\mathbf{C}$; such a manifold will be called the geometric realization of $\mathbf{C}$.

More precisely, to the local isometry class of $\mathbf{M}$, one associates the orbit of the following Cartan triple: let $\mathbf{u}$ be an orthonormal frame at the point $\mathbf{x} \in \mathbf{M}$. The first component of this triple, $\mathfrak{g}=\lambda_{\mathbf{u}}\left(\mathfrak{k}(\mathbf{M})_{\mathbf{x}}\right)$ is the image of the isotropy algebra at the point of $\mathbf{x}$ of the algebra $\mathfrak{k}(M)$ of Killing vector fields of $M$, by the tangent map of the isotropy representation with respect to the frame $\mathbf{u}$. Let $\mathbf{K}$ be a Lie group of Lie algebra $\mathfrak{k}(\mathbf{M})$, and let $\mathbf{H}$ be its connected Lie subgroup of Lie algebra $\mathfrak{k}(\mathbf{M})_{\mathbf{x}}$. If $V$ is an open neighborhood of $1_{\mathbf{K}}$, such that the foliation of $F$ of $V$ determined by the left cosets of $H$, is simple, we showed that $F$ is a reductive Riemannian foliation [10], such that the quotient $V / F$ is locally isometric to $\mathbf{M}[\mathbf{1 3}$, Theorem 1.1].

The $A-S$ torsion of the second component of the Cartan triple is the torsion at $x$, the leaf of $1_{\mathbf{K}}$, of the canonical metric connection of $V / F$, invariant under the local action of $\mathbf{K}$ on $V / F$, induced by left translations. 
The last component of the associated Cartan triple is the $\mathfrak{g}$-part of the Riemannian curvature form with respect to $u$ and to the decomposition of $\mathfrak{o}(n)=\mathfrak{g} \oplus \mathfrak{g}^{\perp}$, evaluated at $\mathbf{x}$.

The $\mathfrak{g}^{\perp}$-component of the Riemannian curvature form with respect to $u, \bar{\Omega}_{\mathfrak{g} \perp}$, may be recovered from this Cartan triple in the following way $[13,(1.24)]$ :

$$
\bar{\Omega}_{\mathfrak{g}^{\perp}}(X, Y)=[\Gamma(X), \Gamma(Y)]_{\mathfrak{g}^{\perp}}+\Gamma(T(X, Y)), \quad \forall X, \quad \forall Y \in \mathbb{R}^{n}
$$

Thus the Riemannian curvature form, with respect to $\mathbf{u}, \Omega=\bar{\Omega}_{\mathfrak{g}} \oplus \bar{\Omega}_{\mathfrak{g}^{\perp}}$, yields the components of the curvature tensor by the usual formulas:

$$
R_{j k r}^{i} e_{i}=\Omega\left(e_{k}, e_{r}\right) e_{j}
$$

Beside the usual metric invariants, such as the Ricci spectrum, an useful invariant is the square norm of the Cartan-Singer map $\Gamma$ of a maximal Cartan triple, given by:

$$
\|\Gamma\|^{2}=\sum_{j=1}^{n} k\left(\Gamma\left(e_{j}\right), \Gamma\left(e_{j}\right)\right) .
$$

In (1.15), $k$ is a fixed multiple of the Killing form.

\section{The classification of 3-dimensional locally homogeneous Riemannian manifolds.}

Let $\left(E_{j}^{i}\right) i, j=\overline{(1, n)}$, be the natural basis of $\mathfrak{g l}(n, \mathbb{R})$, whose elements are acting on $\mathbb{R}^{n}$ by:

$$
E_{j}^{i}\left(e_{k}\right)=\delta_{k}^{i} e_{j}
$$

and let $f_{j}^{i}=E_{j}^{i}-E_{i}^{j}, i>j$, be the natural basis of $\mathfrak{o}(n)$.

In order to describe the moduli space $M_{3} / A_{3}$, as a stratified set, we shall recall first that any proper, nontrivial Lie subalgebra of $\mathfrak{o}(3)$ is conjugated to $\mathfrak{o}(2)=\mathbb{R} f_{1}^{2}$.

Thus there are three types of 3-dimensional Cartan triples: 3-triples $(\mathfrak{o}(3)$, $O, \bar{\Omega}), 1$-triples $(\mathfrak{o}(2), \Gamma, \bar{\Omega})$ and $O$-triples $(O, \Gamma, O)$.

The o(3)-curvature of 3 -triples has the form $\bar{\Omega}=K \cdot \Omega_{1}$,

$$
\Omega_{1}(x, y)=\left(x^{1} y^{2}-x^{1} y^{2}\right) f_{1}^{2}+\left(x^{1} y^{3}-x^{3} y^{1}\right) f_{1}^{3}+\left(x^{2} y^{3}-x^{3} y^{2}\right) f_{2}^{3} .
$$

The geometric realization of such a 3 -triple is a space of constant curvature K. 
Following [3, p. 301], a 1-triple $(\mathfrak{o}(2), \Gamma, \bar{\Omega})$ has the form:

$$
\begin{aligned}
& \Gamma\left(e_{1}\right)=a f_{1}^{3}+b f_{2}^{3}, \\
& \Gamma\left(e_{2}\right)=-b f_{1}^{3}+a f_{2}^{3}, \\
& \Gamma\left(e_{3}\right)=0, \\
& \bar{\Omega}\left(e_{1}, e_{2}\right)=K f_{1}^{2}, \\
& \bar{\Omega}\left(e_{1}, e_{3}\right)=\bar{\Omega}\left(e_{2}, e_{3}\right)=0, \\
& a\left(K+a^{2}+b^{2}\right)=a b=0 .
\end{aligned}
$$

If $a \neq 0$, such a triple is smaller than the triple $\left(\mathfrak{o}(2), O,-a^{2} \Omega_{1}\right)$, and then its geometric realization is a space of constant negative curvature $-a^{2}$.

If $a=0$, the associated Ricci form is:

$$
\rho(X)=\left(K+b^{2}\right)\left(\left(X^{1}\right)^{2}+\left(X^{2}\right)^{2}\right)+2 b^{2}\left(X^{3}\right)^{2}
$$

and the principal Ricci curvatures $\left\{K+b^{2}, 2 b^{2}\right\}$ are those of a space of constant curvature iff $K=b^{2}$. Only in this case our triple is smaller than a 3 -triple and then its geometric realization is a space of constant curvature $b^{2}$.

A 1-triple is maximal iff $a=0, K \neq b^{2} . O(3)$ orbits of such Cartan triples are distinguished by their principal Ricci curvatures, and correspond to the so called Berger metrics [2].

Corollary 2.1. A 3-dimensional l.h.R.s. is locally isometric to a homogeneous space.

Proof. It is enough to notice that all the 1-triples are closed. Other 3dimensional Cartan triples are obviously closed.

A $R-L$ group is a Lie group with a left invariant Riemannian metric. $R-L$ groups may be unimodular or nonunimodular [9].

Corollary 2.2. Assume $\mathbf{M}$ is the geometric realization of a maximal 1triple of Ricci spectrum $\left(K+b^{2}, K+b^{2}, 2 b^{2}\right)$.

a. If $b=0, K>0, \mathbf{M}$ is not isometric to a $R-L$ group.

b. If $b=0, K<0, \mathbf{M}$ is isometric to a nonunimodular $R-L$ group.

c. If $b \neq 0, \mathbf{M}$ is isometric to an unimodular $R-L$ group.

Proof. Let $\mathbf{M}=K / H$ be the geometric maximal 1-triple.

$$
\mathfrak{k}=\operatorname{Span}\left(f_{1}^{2}, e_{1}, e_{2}, e_{3}\right),
$$


has the structure equations:

$$
\begin{aligned}
& {\left[f_{1}^{2}, e_{1}\right]=-e_{2}, \quad\left[f_{1}^{2}, e_{2}\right]=e_{1},} \\
& {\left[f_{1}^{2}, e_{3}\right]=0, \quad\left[e_{1}, e_{3}\right]=b e_{2},} \\
& {\left[e_{1}, e_{2}\right]=-2 b e_{3}\left(K+b^{2}\right) f_{1}^{2}, \quad\left[e_{2}, e_{3}\right]=-b e_{1} .}
\end{aligned}
$$

Assume $\mathbf{M}$ is isometric to a $R-L$ group. Then the Lie algebra $\mathfrak{m}$ of $\mathbf{M}$ has an orthobasis $\left(E_{1}, E_{2}, E_{3}\right)$ with $E_{i}=e_{i}+a_{i} f_{i}^{2}$. Since $\mathrm{m}$ is closed under $[$,$] the following equalities hold:$

$$
a_{2}\left(b+a_{3}\right)=0, \quad a_{1}\left(b+a_{3}\right)=0, \quad 2 b a_{3}-K-b^{2}-a_{1}^{2}-a_{2}^{2}=0 .
$$

a. If $b=0, K>0$, there are no solutions.

b. If $b=0, K<0$, then $a_{2}=a_{3}=0, a_{1}=\sqrt{-\mathbf{K}}$ is a solution, and from (2.4) it follows that this is a nonunimodular $R-L$ group.

c. If $b \neq 0$, then $a_{1}=a_{2}=0, a_{3}=\left(K+b^{2}\right)(2 a b)^{-1}$ is a solution and $\mathfrak{m}$ turns out to be an unimodular $R-L$ group.

The geometric realization of a $O$-triple is a Lie group endowed with a left invariant metric; in this case the Levi-Civita connection is given by $\nabla_{X} Y=\Gamma(X)(Y)$.

It was shown by Milnor [9] that such homogeneous spaces depend at most on 3 parameters. Let $\mathfrak{k}=\mathfrak{k}(O, \Gamma, O)$; two cases are to be distinguished:

If $\mathfrak{k}$ is not unimodular then it has orthonormal basis, $\left(e_{1}, e_{2}, e_{3}\right)$ such that [9]:

$$
\begin{aligned}
& {\left[e_{1}, e_{2}\right]=\alpha e_{2}+\beta e_{3}} \\
& {\left[e_{1}, e_{3}\right]=\gamma e_{2}+\delta e_{3}} \\
& {\left[e_{2}, e_{3}\right]=0, \quad \alpha+\delta>0, \quad \alpha \geq \delta, \quad \beta \geq \gamma, \quad \alpha \gamma+\beta \delta=0 .}
\end{aligned}
$$

Proposition 2.1. There is a one to one onto map from the space of orbits of maximal $O$-triples with nonunimodular associated Lie algebra and the set

$$
N U=((0, \infty) \times(0, \infty) \times(-1,1)) \cup((0, \infty) \times\{0\} \times((-1,0) \cup(0,1))) .
$$

Proof. The basis which satisfies (2.4) brings the Ricci operator to a diagonal form. Let Ricci $\xi$ be defined by $\lambda=\xi \alpha$, then the possibly principal Ricci curvatures expressed in terms of the parameters $(\alpha, \beta, \xi)$, are [9, Lemma 6.5]

$$
\begin{aligned}
\left(r_{11}, r_{22}, r_{33}\right)= & \left(-\left(1+\xi^{2}\right) \alpha^{2}-\frac{1}{2}(1-\xi)^{2} \beta^{2}\right. \\
& -(1+\xi) \alpha^{2}+\frac{1}{2}\left(\xi^{2}-1\right) \beta^{2} \\
& \left.-\xi(1+\xi) a^{2}+\frac{1}{2}\left(1-\xi^{2}\right) \beta^{2}\right),
\end{aligned}
$$


$\alpha>0, \xi \in(-1,1], \beta(1-\xi) \geq 0$. Notice that $r_{11}<0$.

The given triple is smaller than a 1-triple if $r_{11}=r_{22}$ and $r_{33} \geq 0$. This happens iff $\xi=0$. Then, with the previous notation, the 1-triple is given by $b=\frac{1}{2} \beta, K=-\alpha^{2}-\frac{3}{4} \beta^{2}$. From Corollary 2.2 this is possible iff $b=0, K<0$, that is $\beta=0$. Note that by Corollary 3.2.c there are pairs $\left(\mathbf{G}, \mathbf{G}^{\prime}\right)$ of non isometric $R-L$ groups, $\mathbf{G}$ unimodular and $\mathbf{G}^{\prime}$ nonunimodular with $\xi=0, \beta \neq 0$. This contradicts a statement in the last section of [7], there are no such pairs, where $\mathbf{G}$ and $\mathbf{G}^{\prime}$ are both nonunimodular.

The given triple is smaller than a 3 -triple iff $\xi=1$. In this case the geometric realization is a space of constant curvature $-\alpha^{2}$ and we may consider that $\beta=0$.

For the remaining cases, the principal Ricci curvatures in increasing order are: $\left(r_{11}, r_{22}, r_{33}\right)$, if $\xi \in(-1,0)$ and $\left(r_{22}, r_{11}, r_{33}\right)$, if $\xi \in(0,1)$.

The $O(3)$ orbits of two such maximal $O$-triples are different since their sets of principal Ricci curvatures are different.

Now we find the metric correspondent to $(\alpha, \beta, \xi) \in N U$.

Lemma 2.1. Let $A$ be a linear automorphism of $\mathbb{R}^{p}$, and let $v=v^{i} e_{i} \in \mathbb{C}^{p}$ be an eigenvector for the eigenvalue $\lambda$ of $A^{t}$. Suppose $\theta=d x$ is an exact form of an open set $U \subseteq \mathbb{R}^{p}$, and $\omega=\omega^{i} e_{i} \in \mathfrak{D}\left(U, \mathbb{R}^{p}\right)$ is a solution of $d \omega=\theta \wedge A(\omega)$.

Then $\omega_{v}=\exp (-\lambda x) \sum_{i=1}^{p} v^{i} \omega_{i}$ is exact on $U$.

Nonunimodular 3 -dimensional Lie algebras $\mathfrak{k}$ are classified by the determinant $D$ of the ad-action on $[\mathfrak{k}, \mathfrak{k}]$ of some element $x$ of $\mathfrak{k} \backslash[\mathfrak{k}, \mathfrak{k}] . D$ is given in the next formula $[\mathbf{9}$, p. 321$]$ :

$$
D=\frac{4 \xi\left(\alpha^{2}+\beta^{2}\right)}{(1+\xi)^{2} \alpha^{2}}
$$

Lemma 2.1 may be used in order to find a basis of left invariant Pfaff forms $\left(\alpha^{1}, \alpha^{2}, \alpha^{3}\right)$, on the simply connected 3-dimensional Lie nonunimodular $D$ group $\mathbf{K}$ as follows:

Pick up a basis $\left(f_{2}, f_{3}\right)$ of $[\mathfrak{k}, \mathfrak{k}]$, and $f_{1} \in \mathfrak{k} \backslash[\mathfrak{k}, \mathfrak{k}]$. If $\alpha=\alpha^{i} f_{i}$ is the canonical form of $K\left[\mathbf{9}\right.$, p. 321 , first formula], since $\left[f_{2}, f_{3}\right]=0, \alpha^{1}$ is exact, and $\omega=\alpha^{2} f_{2}+\alpha^{3} f_{3}, A=\operatorname{ad} f_{1} \mid[\mathfrak{k}, \mathfrak{k}]$ are verifying the hypothesis of Lemma 2.1. We obtain the solutions:

If $D<1$ and $\lambda_{1}>\lambda_{2}$ are roots of $\lambda^{2}+2 \lambda+D=0$, then:

$$
\begin{aligned}
& \alpha^{1}=d x \\
& \alpha^{2}=-\lambda_{2} \exp \left(\lambda_{1} x\right) d y+\lambda_{1} \exp \left(\lambda_{2} x\right) d z \\
& \alpha^{3}=-\exp \left(\lambda_{1} x\right) d y+\exp \left(\lambda_{2} x\right) d z,(x, y, z) \in \mathbb{R}^{3}=\mathbf{K} .
\end{aligned}
$$


If $D=1$,

$$
\begin{aligned}
& \alpha^{1}=d x \\
& \alpha^{2}=\exp (-x)(x d y+d z), \\
& \alpha^{3}=\exp (-x)((1-x) d y-d z),(x, y, z) \in \mathbb{R}^{3}=\mathbf{K} .
\end{aligned}
$$

If $D>1$ and $(x, y, z) \in \mathbb{R}^{3}=\mathbf{K}$, then:

(2.8) $\alpha^{1}=d x$,

$$
\begin{gathered}
\alpha^{3}=-\exp (-x)(\sin (x \sqrt{D-1}) d y+\cos (x \sqrt{D-1} d z)) \\
\alpha^{2}=\exp (-x)((\sqrt{D-1} \cos (x \sqrt{D-1})+\sin (x \sqrt{D-1})) d y- \\
-(\sqrt{D-1} \sin (x \sqrt{D-1})-\cos (x \sqrt{D-1})) d z) .
\end{gathered}
$$

Let $\theta^{1}, \theta^{2}, \theta^{3}$ be defined in the next formula

$$
\begin{aligned}
& \theta^{1}=\frac{2}{\alpha(1+\xi)} \alpha^{1}, \theta^{2}=\alpha(1+\xi) \alpha^{2}+2(\alpha+\beta \xi) \alpha^{3}, \\
& \theta^{3}=-\alpha(1+\xi) \alpha^{2}+2(\beta-\alpha \xi) \alpha^{3}
\end{aligned}
$$

and $\alpha, \beta . \xi$ and $D$ are constrained by $(2.5)$, then the metric on the $D$ nonunimodular group that corresponds to $(\alpha, \beta, \xi) \in N U$ is

$$
g=\left(\theta^{1}\right)^{2}+\left(\theta^{2}\right)^{2}+\left(\theta^{3}\right)^{2} .
$$

If $\mathfrak{k}$ is unimodular then $\Gamma$ is given by (see $[\mathbf{9}$, p. 319]):

$$
\Gamma\left(e_{1}\right)=-\mu_{1} f_{2}^{3}, \quad \Gamma\left(e_{2}\right)=\mu_{2} f_{1}^{3}, \quad \Gamma\left(e_{3}\right)=-\mu_{3} f_{1}^{2} .
$$

Define the new parameters $\left(\lambda_{i}\right), i=1,2,3$ by

$$
\lambda_{i}=\mu_{1}+\mu_{2}+\mu_{3}-\mu_{i} .
$$

Then the bracket of $\mathfrak{k}$ is $[\mathbf{M i}$, p. 319]:

$$
\begin{aligned}
& {\left[e_{1}, e_{2}\right]=\lambda_{3} e_{3}} \\
& {\left[e_{2}, e_{3}\right]=\lambda_{1} e_{1}} \\
& {\left[e_{3}, e_{1}\right]=\lambda_{2} e_{2}}
\end{aligned}
$$

and the principal Ricci curvatures are:

$$
r_{11}=2 \mu_{2} \mu_{3}, \quad r_{22}=2 \mu_{3} \mu_{1}, \quad r_{33}=2 \mu_{1} \mu_{2} .
$$

The geometric realization of such a nontrivial $O$-triple is one of the following Lie groups:

$$
\tilde{S} L_{2}, \text { the universal covering group of } S L_{2}(\mathbb{R}),
$$


- $\tilde{E}(2)$. the universal covering group of the group of direct isometries of the Euclidean plane,

- $\mathrm{Sol}^{3}$, the universal covering group of the group of direct, time preserving, isometries of the Minkowski plane,

- $N i l^{3}$, the Heisenberg group of $3 \times 3$ real triangular matrices $I_{3}+x E_{1}^{2}+$ $y E_{1}^{3}+z E_{2}^{3}$,

- $\mathbb{S}^{3}$, the group of unit quaternions.

Proposition 2.2. There is one to one and onto map from the set of isometry classes of left invariant metrics on $\tilde{S} L_{2}$ and the set $\wedge$,

$$
\wedge=\left\{\left(\lambda_{1}, \lambda_{2}, \lambda_{3}\right) \in \mathbb{R}^{3}, \lambda_{1} \leq \lambda_{2}<0<\lambda_{3}\right\} .
$$

The parameters are those of (2.12).

Proof. W.l.o.g., one may suppose that $\lambda_{1} \leq \lambda_{2}<0<\lambda_{3}$. From (2.13) it follows that either two of the principal Ricci curvatures are negative and one is positive $\left(\mu_{2} \neq 0\right)$, or there is a double zero principal Ricci curvature and a negative one $\left(\mu_{2}=0\right)$. In the first case the $O$-triple is maximal except for the case when $\lambda_{1}=\lambda_{2}$ when it is smaller then a 1-triple in Corollary 2.2., with the parameters $(b, K)=\left(\frac{1}{2} \lambda_{3},-\frac{3}{4} \lambda_{3}^{2}+\lambda_{1} \lambda_{3}\right)$. In the second, the O-triple is maximal, since by (2.3) if the geometric realization of a maximal 1-triple has a double zero principal Ricci curvature, the other principal Ricci curvature is positive. On the other hand if $\mu_{2}=0$, then by (2.11) and (1.15), $\|\Gamma\|^{2}=\lambda_{1}^{2}+\lambda_{3}^{2}$, and $\|\Gamma\|^{2}$ and the nonzero principal Ricci curvature,

$$
\left(\lambda_{1}, \lambda_{2}=\lambda_{1}+\lambda_{3}, \lambda_{3}\right), \quad \lambda_{1} \leq \lambda_{2}<0<\lambda_{3} .
$$

In order to find the left invariant metric of $\tilde{S} L_{2}$ that corresponds to the parameter $\lambda$, we recall $[14$, p. 462$]$ that this group is the universal covering group of isometries of $H^{2}$. If one identifies $I\left(H^{2}\right)$ with $O H^{2}$ may recover the canonical form of $I\left(H^{2}\right)$ from the fundamental and Levi-Civita connection forms on $O H^{2}$. Therefore we may take the following basis of left invariant Pfaff forms on $\tilde{S} L_{2}\left((x, y, z) \in \mathbb{R}^{3}\right.$ are the coordinates of $\left.\tilde{S} L_{2}\right)$

$$
\begin{aligned}
& \omega^{1}=\cos x d y-\sin x \cosh y d z, \omega^{2}=\sin x d y+\cos x \cosh y d z, \\
& \omega^{3}=d x-\sinh y d z, \quad x, y, z \in \mathbb{R} .
\end{aligned}
$$

Then, the metric associated to the parameter $\lambda \in \Lambda$, is

$$
g_{\lambda}=-\frac{1}{\lambda_{2} \lambda_{3}}\left(\omega^{1}\right)^{2}-\frac{1}{\lambda_{3} \lambda_{1}}\left(\omega^{2}\right)^{2}+\frac{1}{\lambda_{1} \lambda_{2}}\left(\omega^{3}\right)^{2}
$$


Since the proofs of the Propositions 2.3-2.6 do not imply different ideas from those of Propositions 2.1-2.2 (we suggest also the parallel reading of [9]), these proofs shall be omitted.

Proposition 2.3. Any left invariant Riemannian metric on

$$
\mathrm{Sol}^{3}=\left(\left\{e^{z} E_{1}^{1}+e^{-z} E_{2}^{2}+E_{3}^{3}+x E_{1}^{3}+y E_{2}^{3}, x, y, z \in \mathbb{R}\right\}, \cdot\right)
$$

is isometric with one and only one of the following metrics:

$$
\begin{aligned}
& g=\frac{\lambda_{1}}{2}\left(\omega^{1}-\omega^{2}\right)^{2}-\frac{\lambda_{2}}{2}\left(\omega^{1}+\omega^{2}\right)^{2}-\frac{1}{\lambda_{1} \lambda_{2}}\left(\omega^{3}\right)^{2}, \\
& \omega^{1}=e^{z} d y, \omega^{2}=e^{-z} d x, \omega^{3}=d z \\
& \lambda_{1}>0>\lambda_{2} \geq-\lambda_{1} .
\end{aligned}
$$

Proposition 2.4. Any left invariant Riemannian metric on $\tilde{E}(2)=\left(\mathbb{R}^{3}, \otimes\right)$, the universal covering group of the group of motions of the Euclidean plane is isometric with one and only one of the following metrics:

$$
\begin{gathered}
g=\lambda_{1}\left(\omega^{1}\right)^{2}+\lambda_{2}\left(\omega^{2}\right)^{2}+\frac{1}{\lambda_{1} \lambda_{2}}\left(\omega^{3}\right)^{2}, \\
\omega^{1}=(\cos z) d x+(\sin z) d y, \omega^{2}=-(\sin z) d x+(\cos z) d y, \\
\omega^{3}=d z, \lambda_{1}>\lambda_{2}>0 \text { or } \lambda_{1}=\lambda_{2}=1 .
\end{gathered}
$$

Proposition 2.5. The isometry group of any left invariant metric on $\mathrm{Nil}^{3}$ is 4-dimensional. These metrics are homothetic. The nonisometric metrics are determined by a positive parameter $\lambda$, in the formula:

$$
g=\lambda^{2}(d x-y d z)^{2}+d y^{2}+d z^{2} .
$$

Proposition 2.6. There exists a bijection from the set of isometry classes of Riemannian homogeneous metrics on the Euclidean sphere

$$
\mathbb{S}^{3}=\left\{x \in \mathbb{R}^{4}, x=\left(x^{1}, x^{2}, x^{3}, x^{4}\right),\|x\|=1\right\},
$$

to the unbounded simplex $S=\left\{\left(\lambda_{1}, \lambda_{2}, \lambda_{3}\right), \lambda_{1} \geq \lambda_{2} \geq \lambda_{3}>0\right\}$. The metric $g_{\lambda}=4\left(\left(\lambda_{2} \lambda_{3}\right)^{-1} \sigma_{1}^{2}+\left(\lambda_{3} \lambda_{1}\right)^{-1} \sigma_{2}^{2}+\left(\lambda_{1} \lambda_{2}\right)^{-1} \sigma_{3}^{2}\right)$,

$$
\begin{aligned}
& \sigma_{1}=-x^{2} d x^{1}+x^{1} d x^{2}-x^{4} d x^{3}+x^{4} d x^{3} \\
& \sigma_{2}=-x^{3} d x^{1}+x^{4} d x^{2}-x^{1} d x^{3}+x^{2} d x^{4} \\
& \sigma_{3}=-x^{4} d x^{1}+x^{3} d x^{2}-x^{2} d x^{3}+x^{1} d x^{4},
\end{aligned}
$$


is assigned by this bijection to $\lambda \in S$.

The dimension $d_{\lambda}$ of the isometry group of $\left(\mathbb{S}^{3}, g_{\lambda}\right)$ is

$$
d_{\lambda}=\left\{\begin{array}{lll}
3 & \text { if } \quad \lambda_{1}>\lambda_{2}>\lambda_{3} \\
4 & \text { if } \quad \lambda_{1}=\lambda_{2}>\lambda_{3} \text { or } \lambda_{1}>\lambda_{2}=\lambda_{3} . \\
6 & \text { if } \quad \lambda_{1}=\lambda_{2}=\lambda_{3}
\end{array}\right.
$$

Particularly, nonisometric homogeneous structures on $\mathbb{S}^{3}$, depend on 3 real parameters $[\mathbf{1 9}$, p. 352].

Corollary 2.3. Any simple connected 3-dimensional homogeneous Riemannian space is isometric to one and only one of the following:

I. The Riemannian product of round 2-sphere of radius $R, \mathbb{S}_{R}^{2}$ with an Euclidean line,

II. The Riemannian product of a hyperbolic plane $H_{R}^{2}$ of constant curvature - $R^{-2}$ with an Euclidean line,

III. The sphere $\mathbb{S}^{3}$ endowed with one of the metrics $g_{\lambda}$ of (2.18),

IV. $\mathbb{R}^{3}$, endowed with one of the metrics (2.10), (2.14), (2.15), (2.16), (2.17).

The above classification is a concrete exemplification of the general principle [8], that Lie groups with left invariant metrics are "generic" in the set of Riemannian homogeneous spaces of a given dimension.

As a consequence of Corollary 2.3 above we get another proof of the classification of the 1-connected maximal geometries with compact quotient of Thurston $[\mathbf{1 6}, \mathbf{1 4}]$.

We recall that such a geometry is a pair $(X, K)$ where $K$ is a Lie group that acts transitively on $X$, with compact isotropy group, and has a discrete subgroup $\Gamma$, such that $\Gamma^{\backslash K}$ is compact.

Two geometries $(X, K),\left(X^{\prime}, K^{\prime}\right)$ are equivalent is there is a diffeomorphism $f: X \rightarrow X^{\prime}$ that sends the action of $\mathrm{K}$ onto the one of $K^{\prime}$. If $\mathrm{K}$ is subgroup of $K^{\prime}$, and $\operatorname{dim} K<\operatorname{dim} K^{\prime}$, and if the restriction of the action of $K^{\prime}$ to $K$ is still transitive then the geometry of $(X, K)$ is smaller than the geometry of $\left(X, K^{\prime}\right)$; a geometry is maximal, if it is not smaller than another one. The geometry $(X, K)$ is 1-connected, if $X$ is simply connected.

Let us consider that we put on $X$ a metric $g^{\prime}$ such that $K$ acts by isometries (this is possible because the isotropy group is compact). Then $\left(X, g^{\prime}\right)$ is isometric either to $\mathbb{S}_{r}^{2} \times \mathbb{R}$ or with $H_{R}^{2} \times E^{1}$, or to $\left(\mathbb{R}^{3}, g\right)$ where $g$ is one of the metrics $(2.10),(2.14),(2.15),(2.16),(2.17)$, or finally to $\left(\mathbb{S}^{3}, g\right)$ where $g$ is one of the metrics (2.18).

Two geometries in the same family of metrics and based on the same Lie group $K$, are equivalent, since the isomorphism which carries the basis e of 
$k$ corresponding to the first metric onto the basis $e^{\prime}$ corresponding to the second one, induces an equivalence of the two geometries.

It is worth mentioning that the left invariant metrics on $\tilde{S} L_{2}$ depends continuously on the parameter $\lambda \in \Lambda$ of Proposition 2.2. $\Lambda$ is contractible and has an obvious 3-dimensional stratification, such that the 2-dimensional stratum consists of maximal geometries.

Particularly if in Proposition 2.3, $\lambda_{1}=\lambda_{2}=-\lambda_{3}=-1$, we find the metric of $\tilde{S} L_{2}$ given in [16, p. 369]. The nonunimodular $O$-group $(D=0)$, is also a transitive group of isometries of such a geometry.

The set of all left invariant metrics on $\mathrm{Sol}^{3}$ has a structure of a 2-dimensional, contractible stratified set, as shown in Proposition 2.3. For any such geometry, $\mathrm{Sol}^{3}$ is the component of the identity of its group isometries.

The set of all left invariant metrics on $\tilde{\mathrm{E}}(2)$ is also a 2-dimensional stratified set, which may be parametrized by a subset of $\mathbb{R}^{2}$ contractible to the point corresponding to the Euclidean geometry.

$\mathrm{Nil}^{3}$ has a unique left invariant metric, up to a homothety. The isometry group of this geometry is 4-dimensional, and $\mathrm{Nil}^{3}$ is the only 3-dimensional transitive subgroup of this group.

The isometry group of $H_{R}^{2} \times E^{1}$ is 4-dimensional. Although this group has no 3-dimensional transitive Lie subgroup, it is unimodular. Therefore $H^{2} \times E^{1}$ is another geometry of $\mathbb{R}^{3}$.

The isometry group of $H_{R}^{3}$ is 6-dimensional and unimodular. Any proper transitive subgroup of this geometry is a nonunimodular $D$-group, for some $D>1$.

The six contractible families of geometries mentioned above are the only geometries with compact quotient supported by $\mathbb{R}^{3}$.

Two geometries that are members of different families are not equivalent, because the maximal geometries of different families have nonisomorphic groups.

The set of all geometries supported by $\mathbb{S}^{3}$ is a contractible 3-dimensional stratified set, as shown in Proposition 2.6. The round spheres are in the 1-dimensional stratum.

The isometry group of $\mathbb{S}^{2} \times E^{1}$ is unimodular and it does not have a transitive subgroup of dimension 3 . These geometries of $\mathbb{S}^{2} \times \mathbb{R}$, are homothetic, thus equivalent.

As $\mathbb{R}^{3}, \mathbb{S}^{3}$ and $\mathbb{S}^{2} \times \mathbb{R}$ are 1-connected and pairwise nonhomeomorphic, if one chooses one maximal geometry of each of the eight families of geometries encountered above, one obtains all the nonequivalent 3-dimensional, 1-connected geometries with compact quotient.

Notice that $\mathbb{R}^{3}$ also supports geometries without compact quotient, given by its nonunimodular $D$-group structures with left invariant metrics, for 
$D \leq 1, D \neq 0$, as shown by Proposition 2.1.

We mention that a 3-dimensional l.h.R.s. with nontrivial isotropy, is uniquely determined by the Ricci curvature at one point. We would like to know to what extent the geometry is determined by the Ricci form. If one leaves aside products of an elliptic or hyperbolic plane with a line, 3dimensional homogeneous Riemannian manifolds have an $R-L$ group structure. For each algebraic type of 3-dimensional Lie group, from the above metric classification, there is a unique left invariant structure with a given generic Ricci form. Nevertheless, even in the generic case, the Ricci form does not completely determine the algebraic structure of the underlying Lie group; given a Lorentz quadratic form $\rho$ of signature $(-,-,+)$ one may easily find pairs of distinct $R-L$ groups, with the Ricci curvature $\rho$. In the nongeneric case, assume $\rho$ has the form $\rho(X)=\mu\left(\left(X^{1}\right)^{2}+\left(X^{2}\right)^{2}\right)+\nu\left(X^{3}\right)^{2}$. If $\rho$ is the Ricci form of an $R-L$ group, $\nu$ has to be nonnegative. If $\nu>0, \mu<0$, from the proofs of Propositions 2.1, 2.2, and the case $\lambda_{1}>2 \lambda_{2}, \lambda_{2}=\lambda_{3}$ in Proposition 2.6, it follows that there are two unimodular and a nonunimodular $R-L$ group, of Ricci form $\rho$. The case $\nu$ and $\mu$ both positive is uninteresting.

If $\nu=0, \mu \neq 0$, from Corollary 2.2. a.b, it follows that locally, the only homogeneous Riemannian manifold of Ricci form $\rho(X)=\mu\left(\left(X^{1}\right)^{2}+\left(X^{2}\right)^{2}\right)$ is the product of a surface of constant curvature $\mu$ with a line.

Corollary 2.4. For any quadratic form $\rho$ in 3 variables, there are at most finitely many non locally isometric 3-dimensional l.h.R.s., with Ricci form $\rho$, except for the following situations:

i. If $\nu>0$, there is a one-parameter family of nonisometric unimodular $R-L$ structures on $\mathbb{S}^{3}$, with the Ricci form $\rho(X)=\nu\left(X^{3}\right)^{2}$. There is also a unique nonunimodular $R-L$ group, of Ricci form $\rho$.

ii. If $\nu<0$ there are exactly one-parameter family of nonisometric $R-L$ group structures on $\tilde{S} L_{2}$, and $R-L$ group structures on $\mathrm{Sol}^{3}$, whose Ricci form $\rho(X)=\nu\left(X^{3}\right)^{2}$.

Proof. The analysis done in this section points out the following unique $R-L$ groups of nonzero Ricci form $\rho(X)=\nu\left(X^{3}\right)^{2}: \mathbb{S}^{3}$, for $\nu>0$ and $\tilde{S} L_{2}$ and $\mathrm{Sol}^{3}$, for $\nu>0$ and $\tilde{S} L_{2}$ and $\mathrm{Sol}^{3}$ for $\nu<0$.

i. Assume $\nu>0$. Then $\lambda$ in $S$ given in Proposition 2.6 has the components $\left(\lambda_{1}=\lambda_{2}+\lambda_{3}, \lambda_{2}, \lambda_{3}\right), \quad \lambda_{2} \geq \lambda_{3}$ and the square norm of the Cartan-Singer map is $\|\Gamma\|^{2}=\lambda_{2}^{2}+\lambda_{3}^{2}=\lambda_{2}^{2}+\nu^{2} \lambda_{2}^{-2}$. But $\|\Gamma\|^{2}$ is strictly increasing on the domain $[\sqrt{\nu}, \infty)$ of $\lambda_{2}$. If the geometric realizations of two 0 -triples of parameters $\lambda, \lambda^{\prime}$ of this type, even if not maximal, have to have $\|\Gamma\|^{2}=\left\|\Gamma^{\prime}\right\|^{2}$. As such the set of 1-connected homogeneous 3-dimensional manifolds of Ricci form $\rho(X)=\nu\left(X^{3}\right)^{2}$, is $\left\{\mathbf{g}_{\lambda}\right.$ in $\left.(2.18), \lambda=\left(t+\nu t^{-1}, t, \nu t^{-1}\right), t \in[\sqrt{\nu}, \infty)\right\}$. 
ii. Assume $\nu<0$. One may see from the proof of Proposition 2.2, the set of $R-L$ structures on $\tilde{S} L_{2}$, of Ricci form $\rho(X)=\nu\left(X^{3}\right)^{2}$, is $\left\{g_{\lambda}\right.$ in (2.14), $\left.\lambda=\left(\nu t^{-1}, t+\nu t^{-1}, t\right), t \in[\sqrt{-\nu}, \infty)\right\}$

From Proposition 2.3, it follows that only one left invariant metric on $\mathrm{Sol}^{3}$ has the Ricci form $\rho(X)=\nu\left(X^{3}\right)^{2}$.

\section{Metric classification of 4-geometries with 5-dimensional total isometry group.}

Although 4-geometries not necessarily with compact quotient were classified by Wall [18], there are still many things to do toward a complete local metric classification of 4-dimensional 1.h.R.s.-es. Ishihara [4] reconsidered Cartan's original method to list 4-dimensional spaces with nontrivial isotropy. His list was used by Jensen [5], to classify locally, 4-dimensional homogeneous Einstein spaces. Bérgery [2] reobtained and completed Ishihara's list, including the 4-dimensional Lie groups, but his list still had a gap.

In this section we shall given a complete metric classification (i.e., a parametrization of isometry classes) of the 4-geometries that admit a transitive Killing algebra with nontrivial isotopy, and do not admit a de Rham decomposition in factors of constant curvature or of constant holomorphic curvature. By the result of Jensen mentioned above, this happens if the total isometry group of the geometry $M$ is 5-dimensional.

Since we shall use the method of Cartan triples, as presented in the first section, we shall look for maximal Cartan triples $\mathfrak{h}, \Gamma, \bar{\Omega}$, where $\mathfrak{h}$ is a 1 dimensional subalgebra of $\mathfrak{o}(4)$.

Any such subalgebra $\mathfrak{h}$, is conjugated either with $\mathfrak{o}(2)$, or with $\mathbb{R}\left(f_{1}^{2}+m f_{3}^{4}\right)$, with $m \geq 1$.

A Cartan triple $\mathbb{C}=(\mathfrak{o}(2), \Gamma, \bar{\Omega})$ is given by the following data [5]:

$$
\begin{aligned}
\Gamma\left(e_{1}\right) & =a f_{1}^{3}-b f_{2}^{3}+c f_{1}^{4}-d f_{2}^{4} \\
\Gamma\left(e_{2}\right) & =b f_{1}^{3}+a f_{2}^{3}+d f_{1}^{4}+c f_{2}^{4} \\
\Gamma\left(e_{3}\right) & =r f_{3}^{4}, \Gamma\left(e_{4}\right)=t f_{3}^{4} \\
\bar{\Omega}\left(e_{1}, e_{2}\right) & =K_{12} f_{1}^{2} \\
\bar{\Omega}\left(e_{3}, e_{4}\right) & =(t d+r b) f_{1}^{2} \\
\bar{\Omega}\left(e_{1}, e_{3}\right) & =\bar{\Omega}\left(e_{1}, e_{4}\right)=\bar{\Omega}\left(e_{2}, e_{3}\right)=\bar{\Omega}\left(e_{2}, e_{4}\right)=0
\end{aligned}
$$

where the constants $a, b, c, d, r, t, K_{12}$ verify the following relations (see also 
[5, p. 326]):

$$
\begin{aligned}
& 2 a b+d r=0, \quad 2 a d+d t=0, \quad a r+c t=0 \\
& 2 b c-r b=0, \quad 2 c d-t b=0, \\
& t d+r b=0 \\
& a\left(K_{12}+a^{2}+b^{2}+c^{2}+d^{2}\right)=0 \\
& c\left(K_{12}+a^{2}+b^{2}+c^{2}+d^{2}\right)=0 .
\end{aligned}
$$

The discussion of (3.3) splits into the following cases:

Case $b d \neq 0$. Then $a=c=r=t=0$ and the Cartan triple $\mathbb{C}$ is given by:

$$
\begin{aligned}
& \Gamma\left(e_{1}\right)=-b f_{2}^{3}-d f_{2}^{4}, \quad \Gamma\left(e_{2}\right)=b f_{1}^{3}+d f_{1}^{4} \\
& \Gamma\left(e_{3}\right)=\Gamma\left(e_{4}\right)=0, \quad \bar{\Omega}\left(e_{1}, e_{2}\right)=K_{12} f_{1}^{2} \\
& \bar{\Omega}\left(e_{i}, e_{j}\right)=0, \quad \text { if } i<j, \quad,(i, j) \neq(1,2) .
\end{aligned}
$$

Let $R \in S O(4)$ be a rotation by $\theta$ in the plane $\operatorname{Span}\left(e_{3}, e_{4}\right)$ and let $\mathbf{C}^{\prime}=A_{4}(\mathbf{C}, R)$. From (1.10)-(1.12), follows that $\mathbf{C}^{\prime}$ is given by (3.4), depending on the constants $\left(b^{\prime}, d^{\prime}, K_{12}\right)$, where $\left(b^{\prime}, d^{\prime}\right)$ is the image of $(b, d)$, by a rotation with $\theta$ around $(0,0)$ in $\mathbb{R}^{2}$. Therefore any Cartan triple in this case is conjugated to a Cartan triple of the form:

$$
\begin{aligned}
& \Gamma\left(e_{1}\right)=-b f_{2}^{3}, \quad \Gamma\left(e_{2}\right)=b f_{1}^{3}, \quad \Gamma\left(e_{3}\right)=\Gamma\left(e_{4}\right)=0, \\
& \bar{\Omega}\left(e_{1}, e_{2}\right)=K F_{1}^{2}, b>0, \\
& \bar{\Omega}\left(e_{i}, e_{j}\right)=0, \text { if } i<j,(i, j) \neq(1,2) .
\end{aligned}
$$

Case $d=0, b^{2}+d^{2} \neq 0$. In the same manner one may show that such a Cartan triple is conjugated to a Cartan triple defined in (3.5).

If $\mathbf{C}$ is given by (3.5), then the principal Ricci curvatures of the local geometric realization of $\mathbf{C}$ are $0, K+b^{2}, 2 b^{2}$, and it follows that $\mathbf{C}$ is maximal iff $K \neq \pm b^{2}$. If this is the case, it follows that $\mathfrak{k}(\mathbf{C})$ is the Lie algebra of the total group of isometries of the geometric realization $M(\mathbf{C})$ of $\mathbf{C}$. Moreover, due to the discussion of 1-triples in $\S 2$, it follows that $M(\mathbf{C})$ is the product of the geometric realization of the 1-triple defined in (2.2) for $a=0$, with an Euclidean line.

If $\mathbf{C}$ is defined (3.5), from (1.1), (1.2), (1.5)-(1.7) we get the following structure equations of $\mathfrak{k}(\mathbf{C})$ (the null brackets are omitted):

$$
\begin{aligned}
& {\left[e_{1}, e_{2}\right]=2 b e_{3}-\left(K+b^{2}\right) F_{1}^{2}} \\
& {\left[e_{1}, e_{3}\right]=-b e_{2},\left[e_{2}, e_{3}\right]=b e_{1},} \\
& {\left[f^{2}, e_{1}\right]=-e_{2},\left[f_{1}^{2}, e_{2}\right]=e_{1}, b>0 .}
\end{aligned}
$$


The derived algebra $\mathfrak{k}(\mathbf{C})^{\prime}$ is $\operatorname{Span}\left(e_{1}, e_{2}, 2 b e_{3}-\left(K+b^{2}\right) f_{1}^{2}\right)$, and it is isomorphic to:

- $\mathfrak{o}(3)$ if $K+3 b^{2}>0$

- $\quad \mathfrak{s l}(2, \mathbb{R})$, if $K+3 b^{2}<0$,

- the Lie algebra of $\mathrm{Nil}^{3}$, if $K+3 b^{2}=0$.

Thus the corresponding 4-geometries with 5-dimensional group of isometries, described in terms of 3 -geometries are our case geometries of $\mathbb{S}^{3} \times$ $E^{1}, \tilde{S} L_{2} \times E^{1}$ or $\mathrm{Nil}^{3} \times E^{1}$.

Case $b=d=0 \neq K_{12}+a^{2}+c^{2}$. Then $a=c=0$, and one may show that the Cartan triple is not maximal.

Case $b=d=0=K_{12}+a^{2}+c^{2}$. Such a Cartan triple $\mathbf{C}^{\prime}$ is defined by:

$$
\begin{aligned}
& \Gamma^{\prime}\left(e_{1}\right)=a f_{1}^{3}+c f_{1}^{4}, \quad \Gamma^{\prime}\left(e_{2}\right)=a f_{2}^{3}+c f_{2}^{4} \\
& \Gamma^{\prime}\left(e_{3}\right)=r f_{3}^{4}, \quad \Gamma^{\prime}\left(e_{4}\right)=t f_{3}^{4}, \\
& \bar{\Omega}^{\prime}\left(e_{1}, e_{2}\right)=-\left(a^{2}+c^{2}\right) f_{1}^{2}, \quad a r+c t=0, \\
& \bar{\Omega}^{\prime}\left(e_{i}, e_{j}\right)=0, \text { if } i<j, \quad(i, j) \neq(1,2) .
\end{aligned}
$$

Just like in the case $b d \neq 0$, one may act on $\mathbf{C}$ by a suitable rotation in the plane $\operatorname{Span}\left(e_{3}, e_{4}\right)$ to show that $\mathbf{C}^{\prime}$ stays in the orbit of the Cartan triple $\mathbf{C}$ of the form:

$$
\begin{aligned}
& \Gamma\left(e_{1}\right)=c f_{1}^{4}, \Gamma\left(e_{2}\right)=c f_{2}^{4}, \\
& \Gamma\left(e_{3}\right)=r f_{3}^{4}, \Gamma\left(e_{4}\right)=0, \\
& \bar{\Omega}\left(e_{1}, e_{2}\right)=-c^{2} f_{1}^{2}, r \geq 0, \\
& \bar{\Omega}\left(e_{i}, e_{j}\right)=0, \text { if } i<j,(i, j) \neq(1,2) .
\end{aligned}
$$

If $C$ is defined (3.8), we obtain the following structure equations of $\mathfrak{k}(\mathbf{C})$ (the null brackets are omitted):

$$
\begin{aligned}
& {\left[e_{1}, e_{2}\right]=c e_{1},\left[e_{2}, e_{4}\right]=c e_{2} 2,} \\
& {\left[e_{3}, e_{4}\right]=r e_{3},} \\
& {\left[f_{1}^{2}, e_{1}\right]=-e_{2},\left[f_{1}^{2}, e_{2}\right]=e_{1}, r \geq 0 .}
\end{aligned}
$$

The principal Ricci curvatures of the local geometric realization of $\mathbf{C}$ are:

$$
\begin{aligned}
& r_{11}=r_{22}=-2 c^{2}-c r, \\
& r_{33}=-r^{2}-2 c r, \quad r_{44}=-r^{2}-2 c^{2}
\end{aligned}
$$

and from (1.8), (1.9) it follows that $\mathbf{C}$ is maximal if $c \neq r$, or if $c r \neq 0$. If this is the case, it follows that $\mathfrak{k}(\mathbf{C})$ is the Lie algebra of the total group of isometries of the geometric realization $M\left(\mathbf{C}_{-}\right)$of $\mathbf{C}$. Let

$$
C_{-}=\{(r, t), r>0, c>0\}, C_{+}=\{(r, t), r>0, c<0\} .
$$


Suppose $\mathbf{C} \in C_{-} \cup C_{+}$. From (3.9) it follows that the derived algebra of $\mathfrak{k}(\mathbf{C})$, is the normal abelian subalgebra $\mathfrak{n}=\operatorname{Span}\left(e_{1}, e_{2}, e_{3}\right)$. Let $\mathfrak{a}=\mathbb{R} e_{1}$, then $\mathfrak{m}=\mathfrak{n} \oplus \mathfrak{a}$ is a solvable transitive Killing algebra of the $M(\mathbf{C})$, that is a $N C O$-algebra $[\mathbf{1}, \mathbf{1 8}]$ if $\mathbf{C} \in C_{-}$. The diagonal $\Delta$ of $C$ parameterizes spaces of constant negative curvature, and thus, if $\mathbf{C} \in C_{-}, M(\mathbf{C})$ is equivalent with $H^{4}$, in the sense of Thurston.

An analysis of the signs of the principal Ricci curvatures shows that $C_{+} \cup\left(C_{-} \backslash \Delta\right)$ parameterizes the set of isometry classes of the geometric realization of maximal Cartan triples of type (3.8). Since $C_{+}$is contractible, the geometries of $M(\mathbf{C})$, with $\mathbf{C} \in C_{+}$are all equivalent and maximal in the sense of Thurston. One may recognize directly this geometry in Table 1 of [17, p. 122]. If $\mathbf{C} \in C_{+}$, then $M(\mathbf{C})$, is a Lie group and $\left(e_{1}, e_{2}, e_{3}, e_{4}\right)$ is a field of orthonormal frames on it. Let $\left(\theta^{1}, \theta^{2}, \theta^{3}, \theta^{4}\right)$ be the dual coframe. From (3.9), it follows that

$$
\begin{array}{ll}
\theta^{1}=\exp (c t) \cdot d x, & \theta^{2}=\exp (c t) \cdot d y \\
\theta^{3}=\exp (r t) \cdot d z, & \theta^{4}=d t
\end{array}
$$

and then the metric of $M(\mathbf{C})$ is:

$$
g_{c, r}=\exp (2 c t)\left(d x^{2}+d y^{2}\right)+\exp (2 r t) d z^{2}+d t^{2}
$$

which, for $r=-2 c=-2$, is exactly the metric on sol $_{0}^{4}$, implicitly mentioned in $[\mathbf{1 7}$, p. 121].

Suppose now that $\mathfrak{h}=\mathbb{R}\left(f_{1}^{2}-m f_{3}^{4}\right)$, with $m \geq 1$.

$$
\mathfrak{h}^{\perp}=\operatorname{Span}\left(f_{1}^{3}, f_{2}^{3}, f_{1}^{4}, f_{2}^{4}, m f_{1}^{2}-f_{3}^{4}\right) .
$$

Then

$$
\begin{aligned}
& \Gamma\left(e_{2}\right)=a f_{1}^{3}+b f_{2}^{3}+c f_{1}^{4}+d f_{2}^{4}+e\left(m f_{1}^{2}-f_{3}^{4}\right), \\
& \Gamma\left(e_{4}\right)=A f_{1}^{3}+B f_{2}^{3}+C f_{1}^{4}+D f_{2}^{4}+E\left(m f_{1}^{2}-f_{3}^{4}\right),
\end{aligned}
$$

and from the $\mathfrak{h}$-invariance of $\Gamma$ and $\bar{\Omega}$, and since $m \geq 1$, it follows that for $m \neq 2, \Gamma \equiv 0, \bar{\Omega} \equiv 0$, in which case the Cartan triple is not maximal.

Suppose $\underline{m=2}$. Then the $\mathfrak{h}$-invariance of $\Gamma$ and $\bar{\Omega}$, together with (1.3)(1.4), imply that the general form of such a Cartan triple is:

$$
\begin{aligned}
& \Gamma\left(e_{1}\right)=-b\left(f_{1}^{3}+f_{2}^{4}\right)+a\left(f_{2}^{3}-f_{1}^{4}\right), \\
& \Gamma\left(e_{2}\right)=a\left(f_{1}^{3}+f_{2}^{4}\right)+b\left(f_{2}^{3}-f_{1}^{4}\right), \Gamma\left(e_{3}\right)=\Gamma\left(e_{4}\right)=0, \\
& \bar{\Omega}\left(e_{1}, e_{2}\right)=\frac{6}{5}\left(a^{2}+b^{2}\right)\left(f_{1}^{2}+2 f_{3}^{4}\right) \\
& \bar{\Omega}\left(e_{3}, e_{4}\right)=2\left(a^{2}+b^{2}\right)\left(f_{1}^{2}+2 f_{3}^{4}\right) \\
& \bar{\Omega}\left(e_{1}, e_{3}\right)=\bar{\Omega}\left(e_{1}, e_{4}\right)=\bar{\Omega}\left(e_{2}, e_{3}\right)=\bar{\Omega}\left(e_{2}, e_{4}\right)=0,
\end{aligned}
$$


where $a, b$ are constants.

$S O(2)$ acts on the triples (3.13), by rotations in $\operatorname{Span}\left(e_{3}, e_{4}\right)$, and then any such triple is conjugated to a Cartan triple $\mathbf{C}_{a}$ of the form

$$
\begin{aligned}
& \Gamma\left(e_{1}\right)=a\left(f_{2}^{3}-f_{1}^{4}\right), \Gamma\left(e_{2}\right)=a\left(f_{1}^{3}+f_{2}^{4}\right), \\
& \bar{\Omega}\left(e_{1}, e_{2}\right)=\frac{6}{5} a^{2}\left(f_{1}^{2}+2 f_{3}^{4}\right), \Gamma\left(e_{3}\right)=\Gamma\left(e_{4}\right)=0, \\
& \bar{\Omega}\left(e_{3}, e_{4}\right)=2 a^{2}\left(f_{1}^{2}+2 f_{3}^{4}\right), a \geq 0 \\
& \bar{\Omega}\left(e_{1}, e_{3}\right)=\bar{\Omega}\left(e_{1}, e_{4}\right)=\bar{\Omega}\left(e_{2}, e_{3}\right)=\bar{\Omega}\left(e_{2}, e_{4}\right)=0 .
\end{aligned}
$$

From (1.13), (1.14)), we obtain the following expression of the Riemann curvature tensor:

$$
\begin{aligned}
& R_{212}^{1}=2 a^{2}, R_{234}^{1}=-2 a^{2}, R_{434}^{3}=-4 a^{2} \\
& R_{324}^{1}=R_{424}^{2}=R_{313}^{1}=R_{423}^{1}=a^{2} \\
& R_{414}^{1}=R_{323}^{2}=a^{2}
\end{aligned}
$$

which implies that $M\left(\mathbf{C}_{a}\right)$ has the Ricci curvature of a product of a surface of constant curvature $2 a^{2}$ which an hyperbolic plane of curvature $-4 a^{2}$, but it has not the total curvature of such a space, if $a \neq 0$. It is obvious that $\mathbf{C}_{a}$ has not constant holmorphic curvature, since it is not Einstein [5]. Therefore, $\mathbf{C}_{a}$ is maximal if $a>0$. This case was not not included in [5].

Let us set $e_{5}=f_{1}^{2}+2 f_{3}^{4}$. From (1.1), (1.2), (1.5)-(1.7), it follows that the structure equations of $\mathfrak{k}\left(\mathbf{C}_{a}\right)$ are:

$$
\begin{aligned}
& {\left[e_{1}, e_{2}\right]=0,\left[e_{1}, e_{3}\right]=a e_{2},\left[e_{1}, e_{4}\right]=-a e_{1}} \\
& {\left[e_{2}, e_{3}\right]=a e_{1},\left[e_{2}, e_{4}\right]=a e_{2},\left[e_{3}, e_{4}\right]=-2 a^{2} e_{5}} \\
& {\left[e_{1}, e_{5}\right]=e_{2},\left[e_{2}, e_{5}\right]=-e_{1},\left[e_{3}, e_{5}\right]=2 e_{4}} \\
& {\left[e_{4}, e_{5}\right]=-2 e_{3} .}
\end{aligned}
$$

A straightforward computation shows that $\mathfrak{k}\left(\mathbf{C}_{a}\right)$ is isomorphic to the Lie algebra of the semidirect product $\mathbb{R}^{2} \times \mid S L(2, \mathbb{R})$, (look at this algebra as a subalgebra of $\mathfrak{g} l(3, R)$ ).

Therefore, the metrically distinct geometries on $F[\mathbf{1 7}$, p. 122], are the geometric realizations of $\mathbf{C}_{a}, a>0$, and consequently we shall name this geometry $F_{a}^{4}=\left(F^{4}, g_{a}\right)$.

$g_{a}$ is a multiple of the metric given in $[\mathbf{1 7}$, p. 123], but one may find as explained in $\S 1$, in the following way:

Let $\theta=\theta^{i} e_{i}$ be the canonical form of the Lie group $\mathrm{K}$ of Lie algebra $\mathfrak{k}\left(\mathbf{C}_{2}\right)$. Then $\|\theta\|^{2}$ is a transverse metric $[\mathbf{1 0}$, p. 77] of the Riemannian foliation 
defined on $\mathrm{K}$ by $\theta^{2}=0$. From (3.16):

$$
\begin{aligned}
& d \theta^{1}+a \theta^{2} \wedge \theta^{3}-a \theta^{1} \wedge \theta^{4}-\theta^{2} \wedge \theta^{5}=0 \\
& d \theta^{2}+a \theta^{1} \wedge \theta^{3}+a \theta^{2} \wedge \theta^{4}+\theta^{1} \wedge \theta^{5}=0
\end{aligned}
$$

and $\theta^{3}+i \theta^{4}, \theta^{5}$ play the role of the fundamental and connection form of a surface of constant curvature $-4 a^{2}$. Then if we use Poincaré coordinates $x+i y$ in the hyperbolic plane, we get:

$$
\begin{aligned}
& \theta^{3}+i \theta^{4}=(2 a)^{-1} \exp (i t) \cdot \frac{1}{y}(d x+i d y), \\
& \theta^{5}=\frac{1}{2}\left(d t-\frac{d x}{y}\right),
\end{aligned}
$$

and if we put $\theta^{1}+i \theta^{2}=\exp \left(i \frac{t}{2}\right) \cdot(A+i B)$, then from (3.17) $d B-\frac{d y}{2 y} \wedge B=0$, which has the solution

$$
B=\sqrt{y} \cdot d u, \quad \text { and } \quad d A+\frac{d y}{2 y} \wedge A-y_{-}^{-\frac{1}{2}} d x \wedge d u=0,
$$

with the solution

$$
A=y^{-\frac{1}{2}}(x d u+d v),
$$

and eventually the metric on $F^{4}=H^{2} \times \mathbb{R}^{2}$ is

$$
g_{a}=4(a y)^{-2}\left(d x^{2}+d y^{2}\right)+\frac{1}{y}\left((x d u+d v)^{2}+y^{2} d u^{2}\right) .
$$

The following result was essentially discovered by Mostow.

Corollary 3.1. Any l.h.R.s. of dimension 4 is locally isometric to a Riemannian homogeneous space.

Proof. The local geometric realization of a four dimensional Cartan triple $(\mathfrak{h}, \Gamma, \bar{\Omega})$, with $\operatorname{dim} \mathfrak{h} \geqq 2$, is either a space of constant holomorphic curvature, or has a de Rham decomposition in factors of constant curvature [5]. A 1.h.R.s. $M$, that admits a transitive Killing algebra $\mathfrak{k}$, with $\operatorname{dim} \mathfrak{k}=\operatorname{dim} M$, is locally isometric to a Lie group endowed with a left invariant metric. It is easy to remark, from the above list of Cartan triples, that if $(\mathfrak{h}, \Gamma, \bar{\Omega})$ is maximal Cartan triple with $\operatorname{dim} \mathfrak{h}=1$, then $\mathfrak{k}(\mathfrak{h}, \Gamma, \bar{\Omega})$ has a 4-dimensional Lie subalgebras $\mathfrak{g}$, with $\mathfrak{g} \cap \mathfrak{h}=0$.

We obtained also:

Theorem 3.1. Any 4-dimensional geometry in the sense of Thurston, with 5-dimensional total isometry group has either a de Rham decomposition 
$M \times E^{1}$, where $M$ is one and only one of the following 3-dimensional homogeneous Riemannian metrics:

- $\left(\tilde{S} L_{2}, g_{\lambda}\right), \lambda \in \Lambda$ of Proposition 2.2, $\lambda_{1}=\lambda_{2}$, with $g_{\lambda}$ given by (2.14),

- $\left(\mathbb{S}^{3}, g_{\lambda}\right), \lambda \in S$ of Proposition 2.6, $d_{\lambda}=4$.

- $\left(\mathrm{Nil}^{3}, g\right)$ with $g$ given by (2.17),

or is isometric to one and only one of the following de Rham indecomposable Riemannian homogeneous spaces:

- $\left(\mathrm{Sol}_{0}^{4}, g_{c, r}\right)$, with $g_{c, r}$ given by (3.12), $r>0>c$, or $r>0, c>0 r \neq c$,

- $F_{a}^{4}, a>0$.

Remark 3.1. Actually Theorem 3.1 gives a complete metric classification of simply connected homogeneous Riemannian spaces with nontrivial isotropy, since other such spaces either are of constant curvature, or have a de Rham decomposition in factors of constant curvature, or are Kähler surfaces of constant holomorphic curvature [6, Theorem p. 327].

Corollary 3.2 [17, Table 1]. Any maximal geometry with nontrivial isotropy is equivalent to one of the following

\begin{tabular}{|c|c|}
\hline Isotropy & Geometry \\
\hline$S O(4)$ & $S^{4}, E^{4}, H^{4}$ \\
$U(2)$ & $P^{2} \mathbb{C}, H^{2} \mathbb{C}$ \\
$S O(2) \times S O(2)$ & $S^{2} \times S^{2}, S^{2} \times E^{2}, S^{2} \times H^{2}, E^{2} \times H^{2}, H^{2} \times H^{2}$ \\
$S O(3)$ & $S^{3} \times E^{1}, H^{3} \times E^{1}$ \\
$S O(2)$ & $\mathrm{Nil}^{3} \times E^{1}, \mathrm{Sol}_{0}^{4}, \tilde{S} L_{2} \times E^{1}$ \\
$\left(S^{1}\right)_{1,2}$ & $F_{1}^{4}$ \\
\hline
\end{tabular}

Proof. The first four lines correspond to geometries whose total isometry group is at least 6 dimensional, and they follow from [6, Theorem, cases $\mathrm{I}-\mathrm{V}$, p. 327]. Using the same idea as in $\S 2$, one may obtain the remaining lines from Theorem 3.1 .

We end the paper by the remark that, in dimension 5, examples of l.h.R.s. spaces that are not locally isometric to a homogeneous space already exist. Such an example was given by Kowalski [6], and we reprove his result by using Cartan triples, in a natural way.

The idea is to look for nonclosed $n$-dimensional Cartan triples, starting from nonclosed Lie subgroups of $S O(n)$, which is impossible for $n=3$ and $n=4$, due to the Corollary 2.1, 3.1.

Suppose $\underline{n=5}$, and let $\mathfrak{h}=\mathbb{R} f$, where $f=F_{1}^{2}+r f_{3}^{4}$. Then

$$
\mathfrak{h}_{\perp}=\operatorname{Span}\left(r f_{1}^{2}-f_{3}^{4}, f_{1}^{3}, f_{1}^{4}, f_{1}^{5}, f_{2}^{3}, f_{2}^{4}, f_{2}^{5}, f_{3}^{5}, f_{4}^{5}\right) .
$$


After some short computations one finds out that for each pair of reals $(b, d)$, there is a Cartan triple $\mathbf{C}_{b, d}=(\mathfrak{h}, \Gamma, \bar{\Omega})$ defined below:

$$
\begin{array}{ll}
\Gamma\left(e_{1}\right)=b f_{2}^{5}, & \Gamma\left(e_{2}\right)=-b f_{1}^{5}, \\
\Gamma\left(e_{3}\right)=d f_{2}^{5}, & \Gamma\left(e_{4}\right)=-d f_{1}^{5},
\end{array}
$$

$$
\begin{aligned}
& \Gamma\left(e_{5}\right)=0, \\
& \bar{\Omega}\left(e_{1}, e_{2}\right)=-\left(2 r^{-1} b d+b^{2}\left(1+r^{2}\right)^{-1}\right) f \\
& \bar{\Omega}\left(e_{3}, e_{4}\right)=-\left(2 b d+d^{2} r\left(1+r^{2}\right)^{-1}\right) f \\
& \bar{\Omega}\left(e_{i}, e_{j}\right)=0, \quad \text { for other pairs }(i, j) \text { in increasing order. }
\end{aligned}
$$

Proposition 3.1. If $r$ is irrational and $b, d$ are positive numbers such that $b>d>r b$, then the Cartan triple $\mathbf{C}_{b, d}$ is not closed.

Proof. Due to (1.1), (1.2), (1.5) - (1.7), (3.22), it follows that $\mathfrak{k}\left(\mathbf{C}_{b, d}\right)$ is the direct sum of the subalgebras

$$
\begin{aligned}
& \mathfrak{g}_{1}=\operatorname{Span}\left(e_{1}, e_{2}, 2 b\left(d f-e_{5}\right)\right) \\
& \mathfrak{g}_{2}=\operatorname{Span}\left(e_{3}, e_{4}, 2 d\left(b f-e_{5}\right)\right)
\end{aligned}
$$

end each of these subalgebras is isomorphic to $\mathfrak{o}(3)$. Moreover the adjoint action of $f$ on $\mathfrak{k}\left(\mathbf{C}_{b, d}\right)$, with respect to the basis

$$
\left(e_{1}, e_{2}, e_{3}, e_{4}, 2 b\left(d f-e_{5}\right), 2 d\left(b f-e_{5}\right)\right)
$$

has the form:

$$
\left(\begin{array}{ll}
\hline J \\
r
\end{array}\right]_{0}, \text { where } J=\left(\begin{array}{rr}
0 & 1 \\
-1 & 0
\end{array}\right) .
$$

Let $K$ be the simply connected group of Lie algebra $\mathfrak{k}\left(\mathbf{C}_{b, d}\right)$ and let $H$ be the connected subgroup of $K$ tangent to $\mathfrak{h}$. Since ad $(H)$ is nonclosed in the toral subgroup of ad $K$, generated by $\operatorname{Span}\left(F_{1}^{2}, F_{3}^{4}\right)$ and as $K$ is semisimple, it follows that $H$ is not closed in $K$.

\section{References}

[1] R. Azencott and E. Wilson, Homogeneous manifolds with negative curvature I, Trans. Amer. Math. Soc., 215 (1976), 323-362. 
[2] B. Bergery, Les espaces homogènes Riemanniens de dimension 4, Semin. Arthur Besse, Paris, 1978/79, (1981), 40-60.

[3] E. Cartan, Leçons sur la Géométrie des espaces de Riemann, Gauthier - Villars, 1946.

[4] S. Ishihara, Homogeneous Riemannian spaces of four dimensions, J. Math. Soc. Japan, 7 (1955), 345-370.

[5] G. Jensen, Homogeneous Einstein spaces of dimension four, J. Diff. Geom., 3 (1969), 309-349.

[6] O. Kowalski, Counterexample to the second "Singer's Theorem", Ann. Global Anal. Geom., 8 (1990), 211-214.

[7] F. Lastaria, Homogeneous metrics with the same curvature, Simon Stevin, 65 (1991), Number 3-4, 267-281.

[8] F. Lastaria and F. Tricerri, Curvature orbits and locally homogeneous Riemannian manifolds, preprint 1990.

[9] J. Milnor, Curvature of left invariant metrics on Lie proups, Adv. in Math., 21 (1976), 293-329.

[10] P. Molino, Riemannian foliations, Birkhäuser, 1988.

[11] L. Nicolodi and F. Tricerri, On two theorems of I.M. Singer, Ann. Global Anal. Geom., 8 (1990), 193-209.

[12] K. Nomizu, Sur les algèbres de Lie de Générateurs de Killing et l'homogenéité d'une variété riemannienne, Osaka J. Math., 14 (1962), 45-51.

[13] V. Patrangenaru, Locally homogeneous Riemannian manifolds and Cartan triples, Geometriae Dedicata, 50 (1994), 143-164.

[14] P. Scott, The Geometries of 3-manifolds, Bull. London Math. Soc., 15 (1983), 401-487.

[15] F. Tricerri and L. Vanhecke, Homogeneous structures on Riemannian manifolds, London Math. Soc., Lecture Notes, Ser. 83, Cambridge Univ. Press, Cambridge, 1983.

[16] W. Thurston, Three dimensional manifolds, Kleinian Groups and hyperbolic geometry, Bull. of Amer. Math. Soc., (new series), 6 (3), May, 1982, 357-381.

[17] C.T.C. Wall, Geometric structures on compact complex analytic surfaces, Topology, 25 (2) (1986), 119-153.

[18] T.H. Wolter, Homogeneous manifolds with nonpositive curvature operator, Geometriae Dedicata, 37 (1991), 361-370.

[19] W. Ziller, Homogeneous Einstein metrics on Spheres and Projective Spaces, Math. Ann., 259 (1982), 351-358.

Received February 10, 1993.

INDIANA UNIVERSITY

BLOOMINGTON, INDiANA 47405-5701

E-mail address: vpatrang@indiana.edu 
Peng Lin and Richard Rochberg, Trace ideal criteria for Toeplitz and Hankel operators on the weighted Bergman spaces with exponential type

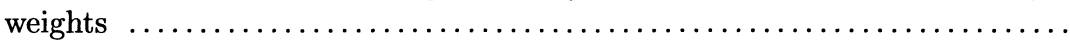

Donald E. Marshall and Arne Stray, Interpolating Blaschke products . .

Kathy D. Merrill and Lynne $\mathbf{H}$. Walling, On quadratic reciprocity over

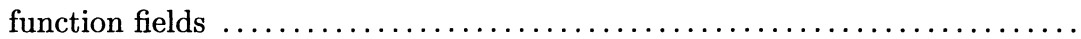

Takahiko Nakazi and Masahiro Yamada, $\left(A_{2}\right)$-conditions and Carleson

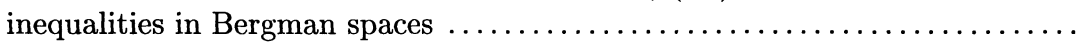

C. Ott, A note on a paper of E. Boasso and A. Larotonda ..............

Victor Patrangenaru, Classifying 3 and 4 dimensional homogeneous Riemannian manifolds by Cartan triples

Carlo Pensavalle and Tim Steger, Tensor products with anisotropic principal series representations of free groups

Ying Shen, On Ricci deformation of a Riemannian metric on manifold with boundary

Albert Jeu-Liang Sheu, The Weyl quantization of Poisson $S U(2) \ldots \ldots$

Alexandra Shlapentokh, Polynomials with a given discriminant over fields of algebraic functions of positive characteristic

Eric Stade and D.I. Wallace, Weyl's law for $S L(3, \mathbb{Z}) \backslash S L(3, \mathbb{R}) / S O(3, \mathbb{R})$

Christopher W. Stark, Resolutions modeled on ternary trees ........ 557

Per Tomter, Minimal hyperspheres in two-point homogeneous spaces .....

Jun Tomiyama, Topological Full groups and structure of normalizers in

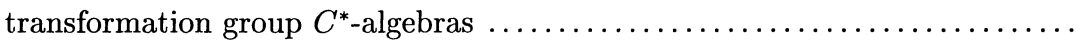

Nik Weaver, Subalgebras of little Lipschitz algebras 


\section{PACIFIC JOURNAL OF MATHEMATICS}

\section{Volume $173 \quad$ No. $2 \quad$ April 1996}

A mean value inequality with applications to Bergman space operators

PATRICK ROBERT AHERn and ZELJKO CUCKOVIC

$H^{p}$-estimates of holomorphic division formulas

MATS ANDERSSON and HASSE CARLSSON

Group structure and maximal division for cubic recursions with a double root

Christian JeAn-Claude Ballot

The Weil representation and Gauss sums

ANTONiA WiLson BLUHER

Duality for the quantum $E(2)$ group

ALFONS VAN DAELE and S. L. WORONOWICZ

Cohomology complex projective space with degree one codimension-two fixed submanifolds 387

KARL HEINZ DOVERMANN and ROBERT D. LITTLE

On the mapping intersection problem

ALEXANDER DRANISHNIKOV

From the $L^{1}$ norms of the complex heat kernels to a Hörmander multiplier theorem for

sub-Laplacians on nilpotent Lie groups

\section{XUAN THINH DUONG}

Isoperimetric inequalities for automorphism groups of free groups

Allen E. Hatcher and Karen Vogtmann

Approximation by normal elements with finite spectra in $C^{*}$-algebras of real rank zero

HUAXIN LIN

Interpolating Blaschke products

DonALD EdDY MARShall and ARNE STRAY

Interpolating Blaschke products generate $H^{\infty}$

JOHN BRADY GARNETT and ARTUR NICOLAU

Classifying 3- and 4-dimensional homogeneous Riemannian manifolds by Cartan triples

VICTOR PATRANGENARU

Polynomials with a given discriminant over fields of algebraic functions of positive

characteristic

ALEXANDRA SHLAPENTOKH

Resolutions modeled on ternary trees

CHRISTOPHER W. STARK

Topological full groups and structure of normalizers in transformation group $C^{*}$-algebras 